\title{
The ADAM graph and its configuration ${ }^{1}$
}

It is well-known that exactly seven of the generalised Petersen graphs are symmetric $(=$ arctransitive), namely the following:

- $G(4,1)$ - the cube graph,

- $G(5,2)$ - the Petersen graph,

- $G(8,3)$ - the Möbius-Kantor graph,

- $G(10,2)$ - the dodecahedron graph,

- $G(10,3)$ - the Desargues graph,

- $G(12,5)$ - the Nauru graph, and

- $G(24,5)$ - the graph that we hereby name the ADAM graph.

Both $G(8,3)$ and $G(10,3)$ are associated with point-line configurations: $G(8,3)$ is the Levi graph (= incidence graph) of the Möbius-Kantor $\left(8_{3}\right)$ configuration, while $G(10,3)$ is the Levi graph if the Desargues $\left(10_{3}\right)$ configuration. A point-circle configuration is called an isometric configuration if all circles have the same radius, and a graph drawn in the plane is called unit-distance graph if all straight edges have the same length.
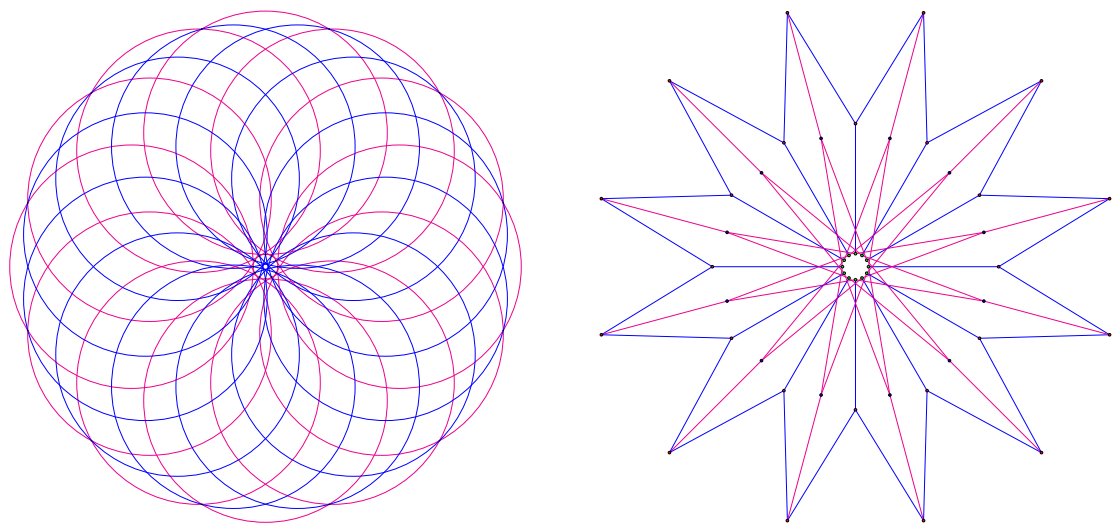

The above figures depict an isometric point-circle configuration $\left(24_{3}\right)$ on the left, whose Levi graph is the generalised Petersen graph $G(24,5)$ drawn as the unit-distance graph on the right. The central detail has been adopted as the logo of our new journal, The Art of Discrete and Applied Mathematics, and because its abbreviation is ADAM, we propose that the generalised Petersen graph $G(24,5)$ and the corresponding $\left(24_{3}\right)$ configuration be called respectively the ADAM graph and the ADAM configuration.

\section{Dragan Marušič and Tomaž Pisanski}

Editors In Chief

\footnotetext{
${ }^{1}$ We would like to thank Nino Bašić and Arjana Žitnik for drawing both figures.
} 